\title{
ALCALOIDES E OUTROS CONSTITUINTES DE Xylopia langsdorffiana (ANNONACEAE)
}

\author{
Marcelo Sobral da Silva,* Josean Fechine Tavares, Karine Formiga Queiroga, Maria de Fátima Agra e José Maria \\ Barbosa Filho \\ Departamento de Ciências Farmacêuticas, Centro de Ciências da Saúde, Universidade Federal da Paraíba, 58051-970 João Pessoa
} - PB, Brasil

Jackson Roberto Guedes da Silva Almeida

Colegiado de Medicina, Universidade Federal do Vale do São Francisco, 56304-205 Petrolina - PE, Brasil

Sâmia Andrícia Souza da Silva

Escola de Enfermagem e Farmácia, Universidade Federal de Alagoas, 57072-970 Maceió - AL, Brasil

Recebido em 10/10/08; aceito em 26/2/09; publicado na web em 3/7/09

\begin{abstract}
ALKALOIDS AND OTHER CONSTITUENTS FROM Xylopia langsdorffiana (ANNONACEAE). The phytochemical investigation of Xylopia langsdorffiana led to the isolation of corytenchine, xylopinine, discretamine, xylopine, ent-atisan-16 $\alpha$-hydroxy-18-oic acid, $13^{2}(S)$ hydroxy-173-ethoxyphaephorbide and quercetin-3- $\alpha$-rhamnoside. Their structures were assigned based on spectroscopic analyses, including two-dimensional NMR techniques. Antioxidant activities of discretamine were measured using the 1,2-diphenyl2-picryl-hydrazyl (DPPH) free radical scavenging assay.
\end{abstract}

Keywords: Xylopia langsdorffiana; alkaloids; atisane diterpene.

\section{INTRODUÇÃO}

A família Annonaceae compreende 2.300 espécies, distribuídas em aproximadamente 130 gêneros. ${ }^{1}$ Sua distribuição geográfica ocorre quase que exclusivamente em regiões tropicais, ${ }^{2}$ sendo que no neotrópico está representada por aproximadamente 40 gêneros e 900 espécies. ${ }^{3}$ Essa família é caracterizada pela presença de terpenoides (principalmente diterpenos), alcaloides (principalmente derivados isoquinolínicos), além de óleos essenciais cuja composição é predominantemente de monoterpenos e sesquiterpenos. ${ }^{4}$

O genero Xylopia é constituído por cerca de 150 espécies, ${ }^{5}$ onde algumas são conhecidas por seus usos etnomedicinais e atividade farmacológica. $X$. aromatica é um agente diurético e utilizada para tratamento de edema de pele, ${ }^{6} X$. frutescens apresentou atividade antimicrobiana ${ }^{7}$ e $X$. cayennensis atividade hipotensora. ${ }^{8}$ Um grande número de componentes químicos tem sido isolado do gênero Xylopia, dentre eles, diterpenos, ${ }^{9}$ sesquiterpenos, ${ }^{10}$ alcaloides ${ }^{11}$ e flavonoides. ${ }^{12}$ Trabalhos realizados por grupos de pesquisas no Brasil com Annonaceae reportaram vários tipos de metabólitos secundários, dentre eles, sesquiterpenos aromadendranos com atividade antifúngica de $X$. brasiliensis, ${ }^{13}$ diterpenos diméricos de $X$. emarginata ${ }^{14}$ e $X$. frutescens, ${ }^{15}$ sesquiterpenos diméricos de $X$. aromatica, ${ }^{16}$ diterpenos traquilobanos e óleos essenciais com atividade acaricida de $X$. serice ${ }^{17}$ e alcaloides com atividade antifúngica de Porcelia macrocarpa. ${ }^{18}$

Xylopia langsdorffiana é uma árvore de 5-7 $\mathrm{m}$ de altura, conhecida popularmente como pimenteira-da-terra. ${ }^{19}$ Em trabalhos publicados sobre essa espécie relataram-se o isolamento, a caracterização estrutural e atividade citotóxica em linhagens de células leucêmicas (V79) e hepatócitos de ratos de um novo diterpeno trachylobano, ${ }^{20}$ além de um novo diterpeno atisano ${ }^{21} \mathrm{e}$, ainda, a caracterização do óleo essencial das folhas que apresentou significativa atividade moluscicida. ${ }^{22}$ Também, foi relatada atividade hipotensora de um diterpeno labdano isolado do caule dessa espécie ${ }^{23}$ e efeito relaxante em traquéia isolada de cobaia. ${ }^{24}$ Por ser considerada uma fonte promissora de substâncias bioativas, continuamos o estudo fitoquímico de X. langsdorffiana e

\footnotetext{
*e-mail: marcelosobral@ltf.ufpb.br
}

neste artigo reportamos o isolamento e a caracterização estrutural de quatro alcaloides, coritenchina (1), xylopinina (2), discretamina (3) e xylopina (4), um diterpeno, o ácido ent-16 $\alpha$-hidroxi-atisan-18-oico (5), um feoforbídeo, o $13^{2}(S)$-hidroxi-17³-etoxifeoforbídeo (6), além do flavonoide glicosilado quercetina-3- $\alpha$-raminosídeo (7). A atividade antioxidante da discretamina frente ao sequestro do radical livre estável 2,2-difenil-1-picril-hidrazil (DPPH) também foi avaliada.

\section{RESULTADOS E DISCUSSÃO}

A substância 1 foi isolada na forma de um pó amorfo. O pico do íon molecular [HR-MS] em $\mathrm{m} / z$ 341,1621 é compatível com a fórmula molecular $\mathrm{C}_{20} \mathrm{H}_{23} \mathrm{NO}_{4}$. No espectro de $\mathrm{RMN}{ }^{13} \mathrm{C}$, observouse a presença de vinte sinais, sendo oito referentes a carbonos não hidrogenados; quatro para carbonos metilênicos; cinco referentes a carbonos metínicos e três sinais para carbonos metoxílicos. Esses dados são compatíveis com esqueleto carbônico de alcalóides tetrahidroprotoberberínicos. ${ }^{25}$

$\mathrm{O}$ espectro de $\mathrm{RMN}{ }^{1} \mathrm{H}$ de $\mathbf{1}$ mostrou na região de hidrogênios em sistemas alifáticos dois dupletos em $\delta_{\mathrm{H}} 3,97$ e $3,73(J=14,6 \mathrm{~Hz})$ correspondentes aos dois hidrogênios em carbono $\mathrm{sp}^{3}$ da posição 8 , compatíveis com substituintes oxigenados nos carbonos C-10 e C-11 de alcalóides pseudotetra-hidroprotoberberínicos. ${ }^{26}$ Além desses, observaram-se também sinais múltiplos entre $\delta_{\mathrm{H}} 2,60-3,20$ referentes aos hidrogênios em sistemas alifáticos. Na região de hidrogênios de sistemas aromáticos, observaram-se quatro simpletos em $\delta_{\mathrm{H}} 6,68$; 6,$61 ; 6,55$ e 6,64 que foram atribuídos aos hidrogênios H-1, H-4, H-9 e H-12, respectivamente. Os três simpletos em $\delta_{\mathrm{H}} 3,84 ; 3,82$ e 3,81 foram atribuídos aos hidrogênios metoxílicos. A localização das metoxilas em C-2, C-3 e C-10 foi feita através de comparação com dados da literatura ${ }^{25}$ e correlações no espectro de NOESY.

$\mathrm{O}$ espectro HMBC mostrou sinais de correlações entre os hidrogênios em $\delta_{\mathrm{H}} 3,97$ e 3,73, com os carbonos em $\delta_{\mathrm{C}} 125,4$ e 59,9 confirmando as atribuições feitas aos hidrogênios $2 \mathrm{H}-8$ e aos carbonos C-12a e C-13a. De maneira análoga, correlação entre o hidrogênio H-1 em $\delta_{H}$ 6,68 com C-13a em $\delta_{C} 59,9$ confirmou a atribuição feita ao hidrogênio H-1. Observaram-se, ainda, correlações entre H-4 em 
$\delta_{\mathrm{H}} 6,61$ com C-2 em $\delta_{\mathrm{C}} 146,1$ e com C-5 em $\delta_{\mathrm{C}} 28,3$, e de H-12 em $\delta_{\mathrm{H}} 6,64$ com C-13 em $\delta_{\mathrm{C}} 36,0$ confirmando a atribuição feita a H-4 e H-12. As demais atribuições são mostradas na Tabela 1. Comparação desses dados de $\mathrm{RMN}{ }^{1} \mathrm{H}$ e ${ }^{13} \mathrm{C}$ com valores da literatura, ${ }^{27}$ permitiu identificar $\mathbf{1}$ como sendo o alcalóide pseudotetra-hidroprotoberberínico coritenchina. Com esses dados de RMN 1D e 2D foi possível reassinalar os deslocamentos químicos dos carbonos C-4 e C-12 publicados na literatura. ${ }^{28}$

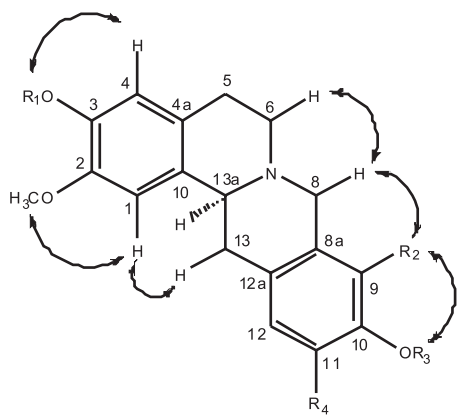

1: $\mathrm{R}_{1}=\mathrm{R}_{3}=\mathrm{CH}_{3} \mathrm{R}_{2}=\mathrm{H}, \mathrm{R}_{4}=\mathrm{OH}$

2: $\mathrm{R}_{1}=\mathrm{R}_{3}=\mathrm{CH}_{3} \mathrm{R}_{2}=\mathrm{H}, \mathrm{R}_{4}=\mathrm{OCH}_{3}$

3: $R_{1}=R_{4}=H, R_{2}=O H, R_{3}=C_{3}$<smiles>COc1ccc2c(c1)C[C@@]1(C)NCCc3cc4c1c(c3-2)OCO4</smiles>

4

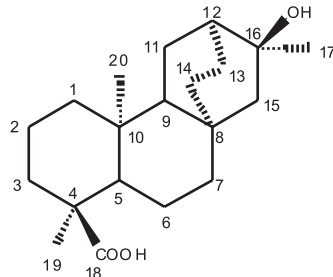<smiles>C=Cc1c(C)c2cc3nc(c4c5[nH]c(cc6nc(cc1[nH]2)C(C)=C6CC)c(C)c5C(=O)[C@]4(C)C(=O)O)[C@@](C)(CCC(=O)OCC)C3(C)C</smiles><smiles>CCOC(Oc1c(-c2ccc(O)c(O)c2)oc2cc(O)cc(O)c2c1=O)C(O)C(O)CO</smiles>

7

Figura 1. Substâncias isoladas de Xylopia langsdorffiana e interações NOE para 1
Tabela 1. Dados de RMN da substância $1\left(500\right.$ MHz) em $\mathrm{CDCl}_{3}$

\begin{tabular}{|c|c|c|c|}
\hline \multirow[b]{2}{*}{$\mathbf{C}$} & \multicolumn{2}{|c|}{ HMQC } & \multirow[b]{2}{*}{ HMBC } \\
\hline & $\delta_{\mathrm{C}}$ & $\delta_{\mathrm{H}}$ & \\
\hline 2 & 146,1 & - & \\
\hline 3 & 144,8 & - & \\
\hline $4 a$ & 126,9 & - & \\
\hline $8 \mathrm{a}$ & 126,0 & - & \\
\hline 10 & 148,2 & - & \\
\hline 11 & 147,9 & - & \\
\hline $12 \mathrm{a}$ & 125,4 & - & \\
\hline $13 b$ & 128,3 & - & \\
\hline \multicolumn{4}{|l|}{ CH } \\
\hline 1 & 108,4 & $6,68(s)$ & C-4a, C-13a \\
\hline 4 & 114,9 & $6,61(\mathrm{~s})$ & C-2, C-5 \\
\hline 9 & 109,3 & $6,55(\mathrm{~s})$ & C-8, C-11, C-12a, \\
\hline 12 & 111,7 & $6,64(\mathrm{~s})$ & C-10, C-13 \\
\hline $13 \mathrm{a}$ & 59,9 & $3,56(\mathrm{~m})$ & $\mathrm{C}-12 \mathrm{a}$ \\
\hline \multicolumn{4}{|l|}{$\mathrm{CH}_{2}$} \\
\hline $5 \mathrm{ax}$ & 28,3 & $2,62(\mathrm{~m})$ & $\mathrm{C}-4, \mathrm{C}-4 \mathrm{a}$ \\
\hline 5 eq & & $3,09(\mathrm{~m})$ & \\
\hline $6 a x$ & 51,4 & $2,64(\mathrm{~m})$ & $\mathrm{C}-4 \mathrm{a}$ \\
\hline 6 eq & & $3,18(\mathrm{~m})$ & \\
\hline $8 \mathrm{ax}$ & 58,0 & $3,97(\mathrm{~d}, J=14,6)$ & C-9, C-12a \\
\hline $8 \mathrm{eq}$ & & $3,73(\mathrm{~d}, J=14,6)$ & \\
\hline $13 \mathrm{ax}$ & 36,0 & $2,84(\mathrm{~m})$ & \\
\hline $13 \mathrm{eq}$ & & $3,20(\mathrm{~m})$ & \\
\hline \multicolumn{4}{|l|}{$\mathrm{OCH}_{3}$} \\
\hline 2 & 56,0 & $3,84(\mathrm{~s})$ & \\
\hline 3 & 55,9 & $3,81(\mathrm{~s})$ & \\
\hline 10 & 55,8 & $3,82(\mathrm{~s})$ & \\
\hline
\end{tabular}

No espectro de COSY, foi possível observar a correlação de H-5 com H-6

No espectro de $\mathrm{RMN}{ }^{13} \mathrm{C}$ de $\mathbf{2}$ observaram-se 21 sinais com padrão de hidrogenação semelhante à substância 1. A ausência de absorção no IV em $3300 \mathrm{~cm}^{-1}$, associada com o surgimento no RMN ${ }^{13} \mathrm{C}$ de um sinal a mais em $\delta_{\mathrm{C}} 55,3$, permitiu identificar 2 como sendo o alcalóide pseudotetra-hidroprotoberberínico tetrametoxilado xylopinina. No espectro de $\mathrm{RMN}{ }^{13} \mathrm{C}$ de $\mathbf{3}$ observaram-se 19 sinais com padrão de hidrogenação também semelhante à $\mathbf{1}$. No espectro de $\mathrm{RMN}{ }^{1} \mathrm{H}$, observaram-se dois simpletos em $\delta_{\mathrm{H}} 7,06$ e 7,03 referentes aos hidrogênios H-1 e H-4 e dois dupletos, $(J=8,4 \mathrm{~Hz})$, em $\delta_{\mathrm{H}}$ 7,17 e 6,98 referentes aos hidrogênios H-11 e H-12, respectivamente. Observou-se também, um dupleto em $\delta_{\mathrm{H}} 4,50(J=15,6$ $\mathrm{Hz}$ ), referente a um dos hidrogênios dos $2 \mathrm{H}-8$, deslocamento compatível com substituintes oxigenados nos carbonos C-9 e C-10 que corresponde a alcaloides tetra-hidroprotoberberínicos. Comparação desses dados de $\mathrm{RMN}{ }^{1} \mathrm{H} \mathrm{e}^{13} \mathrm{C}$ com valores da literatura ${ }^{29}$ permitiu identificar 3 como sendo o alcaloide tetra-hidroprotoberberínico discretamina. Na avaliação da atividade antioxidante deste alcaloide, observou-se que os efeitos dependem da concentração (Tabela 2), tendo uma porcentagem de atividade acima de $90 \%$ em todas 
as concentrações testadas, sendo comparáveis aos padrões ácido ascórbico e quercetina.

Tabela 2. Efeito de sequestro do radical livre DPPH pela discretamina

\begin{tabular}{lccc}
\hline & \multicolumn{3}{c}{ \% Inibição } \\
$\begin{array}{l}\text { Concentração Ácido ascórbico } \\
(\mu \mathrm{g} / \mathrm{mL})\end{array}$ & Quercetina & Discretamina \\
\hline 240 & 99,00 & 98,03 & 94,25 \\
120 & 99,50 & 97,70 & 93,93 \\
60 & 99,00 & 97,67 & 91,51 \\
30 & 99,00 & 97,00 & 90,05 \\
\hline
\end{tabular}

$\mathrm{O}$ espectro $\mathrm{RMN}{ }^{13} \mathrm{C}$ de $\mathbf{4}$ mostrou um total de dezoito sinais. Destes, oito são de carbonos não hidrogenados, cinco de carbonos metínicos, três de carbonos metilênicos e um de carbono metoxílico. $\mathrm{O}$ sinal em $\delta_{\mathrm{C}} 102,7$ sugeriu a presença de um grupamento metilenodioxila. Os dados de $\mathrm{RMN}{ }^{13} \mathrm{C}$ de $\mathbf{4}$ comparados com os da literatura ${ }^{30}$ permitiram propor o esqueleto carbônico de alcalóide aporfínico, com um grupo metilenodioxila e uma metoxila. Os valores de deslocamentos químicos observados no espectro $\mathrm{RMN}{ }^{1} \mathrm{H}$ confirmaram um alcaloide do tipo aporfínico através do deslocamento químico em $\delta_{\mathrm{H}} 8,05$, atribuído a H-11. ${ }^{31}$ Nesse mesmo espectro, observou-se um simpleto em $\delta_{\mathrm{H}} 6,67$ compatível com o hidrogênio H-3 quando as posições 1 e 2 são substituídas. Essa hipótese é corroborada pela presença de dois dupletos em $\delta_{\mathrm{H}} 5,99(\mathrm{~J}=1,0 \mathrm{~Hz})$ e $6,13(\mathrm{~J}=$ $1,0 \mathrm{~Hz}$ ) compatível com substituinte metilenodióxi em C-1 e C-2. Observou-se, ainda, um dupleto em $\delta_{\mathrm{H}} 6,94(\mathrm{~J}=7,0 \mathrm{~Hz})$ atribuído ao hidrogênio $\mathrm{H}-10$. A região de hidrogênios em sistemas alifáticos mostrou dois duplos dupletos, um em $\delta_{\mathrm{H}} 4,46\left(J_{1}=14,2, J_{2}=5,0\right.$ $\mathrm{Hz})$ referente ao hidrogênio $\mathrm{H}-6 \mathrm{a}$ e o outro em $\delta_{\mathrm{H}} 3,10(\mathrm{~J}=5,0 \mathrm{~Hz})$ correspondente ao H-7eq. Além destes, foram observados multipletos em $\delta_{\mathrm{H}} 3,20$ referentes aos hidrogênios $2 \mathrm{H}-5$ e em $\delta_{\mathrm{H}} 2,99$ referentes aos hidrogênios $2 \mathrm{H}-4$. Um sinal em $\delta_{\mathrm{H}} 3,83$ com integração para três hidrogênios foi atribuído à metoxila. Os dados de $\mathrm{RMN}{ }^{13} \mathrm{C}$ foram comparados com os da literatura ${ }^{30}$ e permitiram identificar $\mathbf{4}$ como sendo o alcalóide aporfinico xylopina. Alcaloides aporfínicos são comuns em Annonaceae e têm mostrado atividade citotóxica em linhagens celulares Hep2. ${ }^{32}$

$\mathrm{O}$ espectro de massas [EI-MS] de $\mathbf{5}$ mostrou o pico do íon molecular em $m / z$ 320,24 compatível com a fórmula molecular $\mathrm{C}_{20} \mathrm{H}_{32} \mathrm{O}_{3}$. No espectro infravermelho observaram-se absorções em 3431 e 1696 $\mathrm{cm}^{-1}$, características de grupos hidroxila e carbonila de ácido, respectivamente. O espectro $\mathrm{RMN}{ }^{13} \mathrm{C}$ mostrou três carbonos metílicos, nove carbonos metilênicos, três carbonos metínicos e cinco carbonos não hidrogenados. No espectro $\mathrm{RMN}{ }^{1} \mathrm{H}$ observou-se a presença de metilas terciárias em $\delta_{\mathrm{H}} 1,28 ; 1,16$ e 0,97 . A análise desses dados caracterizou 5 como um diterpeno tetracíclico com esqueleto ent-atisano ou entkaurano com grupo hidroxila em C-16 e grupo carboxila em C-18. ${ }^{33}$ O deslocamento químico de $\mathrm{C}-16 \mathrm{em} \mathbf{5}\left(\delta_{\mathrm{C}} 71,10\right)$ foi condizente com diterpenos ent-atisano-16-hidroxi. ${ }^{34}$ Assim, 5 é o ácido ent-atisano- $16 \alpha$ hidroxi-18-oico, ${ }^{35}$ mas com atribuição inequívoca dos deslocamentos químicos dos carbonos $\mathrm{C}-1$ e C-7 detectados por correlações observadas no $\mathrm{HMBC}$ dos sinais em $\delta_{\mathrm{H}} 1,03\left(\mathrm{CH}_{3}-20\right)$ com o carbono em $\delta_{\mathrm{C}} 38,4$ $(\mathrm{C}-1)$ e $\delta_{\mathrm{H}} 0,94(\mathrm{H}-1)$ com o carbono em $\delta_{\mathrm{C}} 13,5\left(\mathrm{CH}_{3}-20\right)$. As demais atribuições estão compiladas na Tabela 3 . O espectro de COSY mostrou sinais de correlações entre H-3 e H-6 e H-13 com H-14.

$\mathrm{O}$ espectro RMN ${ }^{1} \mathrm{H}$ de $\mathbf{6}$ revelou absorções para um grupo de hidrogênios vinílicos em $\delta_{\mathrm{H}} 7,93\left(\mathrm{dd}, J_{1}=17,8\right.$ e $\left.J_{2}=11,6 \mathrm{~Hz}\right), 6,24$ (dd, $J_{1}=17,8$ e $\left.J_{2}=1,6 \mathrm{~Hz}\right), 6,14\left(\mathrm{dd}, J_{1}=11,6\right.$ e $\left.J_{2}=1,6 \mathrm{~Hz}\right)$, três metilas olefínicas em $\delta_{\mathrm{H}} 3,37,3,16$ e 3,65 e três hidrogênios olefínicos
Tabela 3. Dados de RMN da substância 5 (500 MHz) em $\mathrm{CDCl}_{3}$

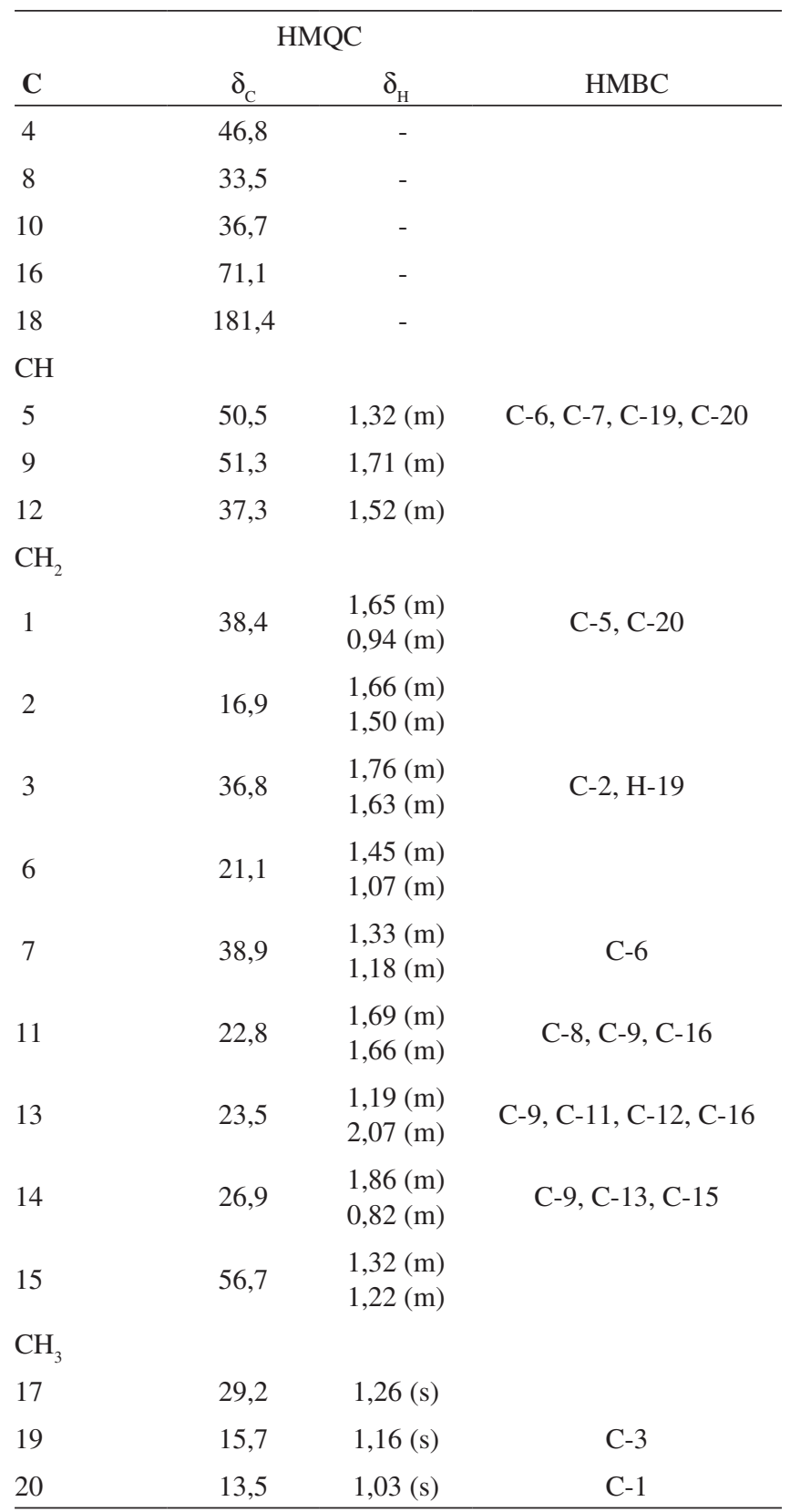

em $\delta_{H}$ 9,30, 9,45 e 8,53. O espectro de IV mostrou absorção em 3397 $\mathrm{cm}^{-1}$, referente à deformação axial de $\mathrm{N}-\mathrm{H}$, e em $1612 \mathrm{~cm}^{-1}$, relativa à deformação de ligação dupla em sistemas conjugados. As bandas em 1731 e $1695 \mathrm{~cm}^{-1}$ foram atribuídas a grupos carbonílicos. Esses dados foram condizentes com núcleo porfirínico de feofitinas. Os dados de $\mathrm{RMN}{ }^{1} \mathrm{He}{ }^{13} \mathrm{C}$ de $\mathbf{6}$ foram semelhantes aos dados publicados na literatura, ${ }^{36}$ porém, a ausência do sinal em $\delta_{\mathrm{C}} 64,6$ e o surgimento do sinal de carbono não hidrogenado ligado a oxigênio em $\delta_{C} 89,2$ permitiram sugerir a inserção de uma hidroxila na posição C-13. ${ }^{2}$ Através da comparação desses dados de $\mathrm{RMN}{ }^{1} \mathrm{H}$ e ${ }^{13} \mathrm{C}$ com valores descritos na literatura, ${ }^{37}$ foi possível identificar $\mathbf{6}$ como sendo $13^{2}$ (S)-hidroxi-1733-etoxifeoforbídeo.

A quercetina-3- $\alpha$-raminosídeo 7 foi identificada através da comparação direta dos dados espectroscópicos obtidos com os valores descritos na literatura. ${ }^{38}$

Todas as substâncias estão sendo relatadas pela primeira vez na espécie em estudo, sendo que $\mathbf{1}$ e $\mathbf{5}$ tiveram dados de carbono e hidrogênio assinalados inequivocamente e $\mathbf{6}$ está sendo relatado pela 
primeira vez como produto de planta. Dessa forma, os resultados contribuem para a quimiotaxonomia de Annonaceae, em especial, do gênero Xylopia.

\section{PARTE EXPERIMENTAL}

\section{Procedimentos experimentais gerais}

Os espectros de absorção na região do infravermelho foram obtidos em aparelho Bomem modelo MB 100M em pastilhas de $\mathrm{KBr}$. Os espectros de massas foram obtidos por inserção direta e energia de ionização 70 eV em espectrômetros VG Autospec e Hewlett Packard 5971. Os espectros de RMN foram obtidos em espectrômetro Mercury-Varian a $200\left[200 \mathrm{MHz}\left({ }^{1} \mathrm{H}\right)\right.$ e $\left.50 \mathrm{MHz}\left({ }^{13} \mathrm{C}\right)\right]$ (LTF/UFPB) e Brucker-Ac $500\left[500 \mathrm{MHz}\left({ }^{1} \mathrm{H}\right)\right.$ e $\left.125 \mathrm{MHz}\left({ }^{13} \mathrm{C}\right)\right]$ (CENAUREM/ UFC). Os solventes empregados foram $\mathrm{CDCl}_{3}, \mathrm{CD}_{3} \mathrm{OD}$ e $\mathrm{C}_{5} \mathrm{D}_{5} \mathrm{~N}$ cujos picos característicos em $\mathrm{RMN}{ }^{1} \mathrm{He} \mathrm{e}^{13} \mathrm{C}$ serviram para ajuste de escala de frequência. Para as cromatografias em coluna utilizou-se como fase estacionária sílica gel 60 (70-230 Mesh) da Merck, óxido de alumínio 90 Seg. Brockman e Sephadex LH-20 (Pharmacia, Uppsala-Sweden). A cromatografia em camada delgada comparativa (CCDC) foi empregada para análise e reunião das frações obtidas por cromatografia em coluna. Foram utilizadas placas de vidro cuja fase fixa foi preparada com uma suspensão de sílica gel PF254 7749 (Merck) em água. As substâncias em análise foram evidenciadas pelo uso de radiação ultravioleta sob os comprimentos de onda de 254 e $366 \mathrm{~nm}$ e pela impregnação das placas em cubas de vidro saturadas por vapores de iodo e reagente de Dragendorff.

\section{Material vegetal}

As folhas e o caule de Xylopia langsdorffiana foram coletados no município de Cruz do Espírito Santo, estado da Paraíba, em julho de 2002. O material botânico foi identificado pela Prof ${ }^{a}$ M. de F. Agra, do setor de botânica do Laboratório de Tecnologia Farmacêutica (LTF); uma exsicata da planta está depositada no Herbário Prof. Lauro Pires Xavier (JPB), da Universidade Federal da Paraíba, sob identificação AGRA 5541.

\section{Procedimento para obtenção dos extratos e isolamento dos constituintes}

O pó do caule $(4100 \mathrm{~g})$ seco e pulverizado foi submetido à maceração com etanol a $95 \%$ e hidróxido de amônia $\left(\mathrm{NH}_{4} \mathrm{OH}\right)$ a $5 \%$ durante 72 h. Após exaustiva extração a solução extrativa foi concentrada em rotavapor sob pressão reduzida a temperatura de $45{ }^{\circ} \mathrm{C}$, obtendo-se o extrato etanólico bruto denominado EEB (300 g).

O EEB foi tratado com uma solução de ácido clorídrico a $3 \%$ sob agitação mecânica e filtrado em celite, fornecendo um precipitado e uma solução ácida que foi extraída com clorofórmio (3 x 300 mL) obtendo-se a fase clorofórmica I (7,0 g). A fase aquosa ácida foi alcalinizada, a frio, com uma solução de hidróxido de amônio até $\mathrm{pH}$ 9,0-10,0 e re-extraída com clorofórmio obtendo-se a fase clorofórmica II (4,0 g) que foi denominado de Fração dos Alcaloides Terciários Totais (FAT). A FAT foi submetida à cromatografia em coluna (CC) utilizando como adsorvente alumina e como eluentes, hexano, clorofórmio e metanol puros ou em misturas binárias em gradiente crescente de polaridade obtendo-se 45 frações de $100 \mathrm{~mL}$ cada. As 45 frações foram monitoradas através de cromatografia em camada delgada comparativa (CCDC) em diferentes sistemas de solventes e reunidas em grupos de frações. As frações FAT-9-12, FAT-15-17, FAT-19-24 e FAT-37-38 foram submetidas à cromatografia em camada delgada preparativa (CCDP) utilizando como fase estacionária sílica gel 60 e como fase móvel $\mathrm{CHCl}_{3} / \mathrm{MeOH}$ (99:1) obtendo-se 1 (10 mg), 2 (25 mg), 3 (20 mg) e 4 (30 mg).

$\mathrm{O}$ pó das folhas seco e pulverizado $(4000 \mathrm{~g})$ foi submetido à maceração com EtOH a 95\%. O extrato resultante foi particionado com hexano, clorofórmio e acetato de etila. $\mathrm{O}$ extrato clorofórmico foi submetido à cromatografia em coluna com sílica gel e eluída com hexano e acetato de etila em gradiente crescente de polaridade e recolheram-se 80 frações de $100 \mathrm{~mL}$ cada. As 80 frações foram monitoradas por CCDC e reunidas. A fração 10-12 foi recristalizada com metanol obtendo-se 5 (40 mg). A fração 15-17 foi recromatografada em coluna com sílica gel e eluída com hexano e acetato de etila em gradiente crescente de polaridade, obtendo-se 6 (15 mg). A fração 65 foi filtrada em Sephadex LH-20 com metanol obtendo-se 7 (12 mg).

\section{Avaliação da atividade antioxidante da discretamina frente ao sequestro do radical livre 2,2-difenil-1-picril-hidrazil (DPPH)}

Foram preparadas uma solução de DPPH (Sigma) $45 \mu \mathrm{g} / \mathrm{mL}$ e soluções com a substância teste e padrões em 4 concentrações diferentes $(240,120,60$ e $30 \mu \mathrm{mol} / \mathrm{L}) \mathrm{em} \mathrm{MeOH}$. As atividades antioxidantes das substâncias testadas foram comparadas com aquelas dos padrões ácido L-(+)-ascórbico (Merck) e quercetina (Sigma), e o declínio da concentração do radical foi monitorado por espectrofotometria no visível em $\lambda=517 \mathrm{~nm}$, após 15 min. ${ }^{39}$ As medidas de absorbância foram feitas em espectrofotômetro Spectronic ${ }^{\circledR} 20$ Genesys. O experimento foi feito em triplicata e a absorção média foi anotada para cada concentração. O mesmo procedimento foi realizado para os controles positivos (ácido ascórbico e quercetina).

Xylopinina, 2: $\mathrm{RMN}^{1} \mathrm{H}\left(200 \mathrm{MHz}, \mathrm{CDCl}_{3}\right)$ : 2,60 ( $\left.m, \mathrm{H}-5 \mathrm{ax}\right), 2,62$ ( $m$, H-5eq), 2,67 ( $m$, H-6ax), 2,87 ( $m$, H-13ax), 3,14 ( $m$, H-6eq), 3,28 ( $m$, H-13eq), 3,56 ( $m, \mathrm{H}-13 \mathrm{a}), 3,61$ ( $s l \mathrm{H}-8 \mathrm{ax}, \mathrm{H}-8 \mathrm{eq}), 3,84$ ( $s, 11-$ $\left.\mathrm{OCH}_{3}\right), 3,83\left(s, 10-\mathrm{OCH}_{3}\right), 3,82\left(s, 3-\mathrm{OCH}_{3}\right), 3,87\left(s, 2-\mathrm{OCH}_{3}\right) 6,55(s$, $\mathrm{H}-9), 6,59(s, \mathrm{H}-4), 6,64(s, \mathrm{H}-12) 6,72(s, \mathrm{H}-1)$. RMN ${ }^{13} \mathrm{C}(50 \mathrm{MHz}$, $\left.\mathrm{CDCl}_{3}\right): 147,3$ (C-2), 147,3 (C-3), 126,6 (C-4a), 126,2 (C-8a), 147,2 (C-10), 147,5 (C-11), 126,2 (C-12a), 129,7 (C-13b), 108,3 (CH-1), 112,2 (CH-4), 108,8 (CH-9), 112,2 (CH-12), 59,5 (CH-13a), 28,6 $\left(\mathrm{CH}_{2}-5\right), 51,3\left(\mathrm{CH}_{2}-6\right), 58,2\left(\mathrm{CH}_{2}-8\right), 36,4\left(\mathrm{CH}_{2}-13\right), 55,8\left(\mathrm{H}_{3} \mathrm{CO}-3\right)$, $55,8\left(\mathrm{H}_{3} \mathrm{CO}-10\right), 55,7\left(\mathrm{H}_{3} \mathrm{CO}-11\right), 55,9\left(\mathrm{H}_{3} \mathrm{CO}-2\right)$.

Discretamina 3: $\mathrm{RMN}{ }^{1} \mathrm{H}\left(200 \mathrm{MHz}, \mathrm{C}_{5} \mathrm{D}_{5} \mathrm{~N}\right): 2,57$ ( $\left.m, \mathrm{H}-5 \mathrm{ax}\right)$, 2,65 ( $m, \mathrm{H}-6 \mathrm{ax}), 3,14$ ( $m, \mathrm{H}-5 \mathrm{eq}), 3,17$ ( $m, \mathrm{H}-6 \mathrm{eq}), 3,47$ ( $m, \mathrm{H}-13 \mathrm{eq})$, $4,10$ ( $m, \mathrm{H}-13 \mathrm{a}), 4,50$ ( $d, \mathrm{H}-8 \mathrm{eq}), 3,64$ ( $m, \mathrm{H}-8 \mathrm{ax}), 3,82\left(s, 2-\mathrm{OCH}_{3}\right)$, 3,94 ( $\left.s, 9-\mathrm{OCH}_{3}\right), 6,98$ ( $\left.d, J=8,4, \mathrm{H}-12\right), 7,06(s, \mathrm{H}-4), 7,03(s, \mathrm{H}-1)$, $7,17(d, J=8,4, \mathrm{H}-11)$. RMN ${ }^{13} \mathrm{C}\left(50 \mathrm{MHz}, \mathrm{C}_{5} \mathrm{D}_{5} \mathrm{~N}\right): .147,3(\mathrm{C}-2), 146,5$ (C-3), 127,9 (C-4a), 126,9 (C-8a), 144,8 (C-10), 148,9 (C-11), 129,3 (C-12a), 129,4 (C-13b), 110,0 (CH-1), 116,3 (CH-4), 115,9 (CH-9), 124,6 (CH-12), 60,0 (CH-13a), 29,4 ( $\left.\mathrm{CH}_{2}-5\right), 51,9\left(\mathrm{CH}_{2}-6\right), 54,7$ $\left(\mathrm{CH}_{2}-8\right), 37,0\left(\mathrm{CH}_{2}-13\right), 56,1\left(\mathrm{H}_{3} \mathrm{CO}-2\right), 59,7\left(\mathrm{H}_{3} \mathrm{CO}-9\right)$.

Xylopina 4: $\mathrm{RMN}{ }^{1} \mathrm{H}\left(200 \mathrm{MHz}, \mathrm{CD}_{3} \mathrm{OD}\right): 2,99$ ( $\left.m, \mathrm{H}-4 \mathrm{ax}\right)$, 3,10 (dd, $J=5$, H-7eq), 3,2 ( $m$, H-5ax), 3,69 ( $m$, H-5eq), 4,38 ( $d d$, $J=14,2$; $5 \mathrm{~Hz}, \mathrm{H}-6 \mathrm{a}), 5,99$ (d, J=1,0 Hz, $6,13\left(d, J=1,0 \mathrm{~Hz}, \mathrm{OCH}_{2} \mathrm{O}\right)$, 6,67 ( $s, \mathrm{H}-3), 6,92$ ( $s, \mathrm{H}-8), 6,94$ ( $d, J=7, \mathrm{H}-10), 8,05$ ( $d, J=7, \mathrm{H}-11$ ), $3,83\left(s, 9-\mathrm{OCH}_{3}\right) . \mathrm{RMN}^{13} \mathrm{C}\left(50 \mathrm{MHz}, \mathrm{CD}_{3} \mathrm{OD}\right): 144,2$ (C-1), 144,9 (C-2), 125,3 (C-3a), 139,9 (C-7a), 161,0 (C-9), 124,1 (C-11a), 117,3 (C-11b), 121,2 (C-11c), 107,8 (CH-3), 54,2 (CH-6a), 114,8 (CH-8), 114,1 (CH-10), 129,9 (CH-11), 26,3 ( $\left.\mathrm{CH}_{2}-4\right), 42,8\left(\mathrm{CH}_{2}-5\right), 34,6$ $\left(\mathrm{CH}_{2}-7\right), 102,7\left(\mathrm{OCH}_{2} \mathrm{O}-1,2\right) 55,8\left(\mathrm{H}_{3} \mathrm{CO}-9\right)$.

$13^{2}$ (S)-hidroxi-173-etoxifeoforbídeo 6: $\mathrm{RMN}^{1} \mathrm{H}\left(500 \mathrm{MHz}^{3} \mathrm{CDCl}_{3}\right)$ : $8,0\left(\mathrm{dd}, J_{1}=17,7 ; J_{2}=11,4 \mathrm{~Hz}, \mathrm{H}-3 \mathrm{a}\right), 9,46$ (s, H-5), 9,61 (s, H-10), 4,18 
(m, H-17), 4,52 (m, H-18), 8,66 (s, H-20), 6,30 (dd, J = 17,7 Hz 1H-3b), 6,19 (dd, $J=11,4 \mathrm{~Hz} 1 \mathrm{H}-3 \mathrm{~b}$ ), 3,72 (q, $J=7,2 \mathrm{~Hz} 2 \mathrm{H}-8 \mathrm{a}$ ), 2,94; 2,53 (m, 2H-17a), 2,29 (m, 2H-17b), 4,11 (q, $J=7,2 \mathrm{~Hz} 2 \mathrm{H}-17 \mathrm{~d}$ ), 3,24 (s, $3 \mathrm{H}-7 \mathrm{a}), 1,71$ (t, $J=7,7 \mathrm{~Hz} 3 \mathrm{H}-8 \mathrm{~b}), 3,63$ (s, 3H-12a), 1,16 (t, $J=7,2 \mathrm{~Hz}$ $3 \mathrm{H}-17 \mathrm{e}$ ), 1,62 (d, $J=7,2 \mathrm{~Hz} 3 \mathrm{H}-18 \mathrm{a}), 3,74$ (s, $\mathrm{OCH}_{3}-13 \mathrm{~d}$ ). $\mathrm{RMN}{ }^{13} \mathrm{C}$ (125MHz, $\left.\mathrm{CDCl}_{3}\right): 142,3$ (C-1), 132,0 (C-2), 136,5 (C-3), 136,6 (C-4), 155,0 (C-6), 136,6 (C-7), 145,3 (C-8), 150,1 (C-9), 138,0 (C-11), 129,8 (C-12), 127,2 (C-13), 192,2 (C-13'), 89, 2 (C-132), 172,7 (C-13 $\left.{ }^{3}\right), 150,1$ (C-14), 104,4 (C-15), 162,8 (C-16), 173,8 (C-173), 173,0 (C-19), 129, 2 $\left(\mathrm{CH}-3^{1}\right), 98,1$ (CH-5), 104,4 (CH-10), 52,0 (CH-17), 50,5 (CH-18), 94,0 $(\mathrm{CH}-20), 123,1\left(\mathrm{CH}_{2}-3^{2}\right), 19,7\left(\mathrm{CH}_{2}-8^{1}\right), 31,8\left(\mathrm{CH}_{2}-17^{1}\right), 31,3\left(\mathrm{CH}_{2}-17^{2}\right)$, 60,7 $\left(\mathrm{CH}_{2}-17^{4}\right), 17,6\left(\mathrm{CH}_{3}-8^{2}\right), 14,3\left(\mathrm{CH}_{3}-17^{5}\right), 22,9\left(\mathrm{CH}_{3}-18^{1}\right), 12,3$ $\left(\mathrm{CH}_{3}-2^{1}\right), 11,4\left(\mathrm{CH}_{3}-7^{1}\right), 12,6\left(\mathrm{CH}_{3}-12^{1}\right), 53,6\left(\mathrm{H}_{3} \mathrm{CO}-13^{4}\right)$.

\section{AGRADECIMENTOS}

Ao CNPq pelo apoio financeiro, a V. C. de O. Costa (LTF/UFPB) e a D. Esdras (CENAUREM/UFC) pela obtenção dos espectros de RMN de 200 e 500 MHz.

\section{REFERÊNCIAS}

1. Hutchinson, J.; The families of flowering plants, Clarendon Press: Oxford, 1973, p. 22-168; Maas, P. J. M.; Kamer, H. M.; Junikka, L.; Mello-Silva, R. D.; Rainer, H.; Rodriguésia 2001, 65, 98.

2. Heywood, V. H.; Flowering plants of the world, Oxford University Press: Oxford, 1985

3. Chatrou, L. W.; Rainer, H.; Maas, P. J. M. Em Annonaceae (Soursop Family); Smith, N.; Mori, S. A.; Henderson, A.; Stevenson, D. W.; Heald, S. V., eds.; Princeton University Press: New Jersey, 2004, p. 18.

4. Leboeuf, M.; Cavé, A.; Bhaumik, P. K.; Mukherjee, B.; Mukherjee, R.; Phytochemistry 1982, 21, 2783.

5. Brummitt, R. K.; Vascular Plant - Families and Genera, Royal Botanic Gardens: Kew, 1992.

6. Takarashi, J. A.; Pereira, C. R.; Pimenta, L. P. S.; Boaventura, M. A. D.; Silva, L. G. F. E.; Nat. Prod. Res. 2006, 20, 21.

7. Macêdo, M.; Ferreira, A. R.; Rev. Bras. Farmacogn. 2004, 14, 40.

8. Nascimento, A. A.; Ribeiro, E. A. N.; Oliveira, J. M.; Medeiros, F. A.; Silva, M. S.; Medeiros, I. A.; Rev. Bras. Farmacogn. 2006, 16, 17.

9. Andrade, N. C.; Barbosa-Filho, J. M.; Agra, M. F.; Da-Cunha, E. V. L.; Silva, M. S.; Recent. Res. Devel. Phytochem. 2003, 7, 1.

10 Moreira, I. C.; Roque, N. F.; Contini, K.; Lago, J. H. G.; Rev. Bras. Farmacogn. 2007, 17, 55.

11. Harrigan, G. G.; Gunatilaka, A. A. L.; Kingston, D. G. I.; Chan, G. W.; Johnson, R. K.; J. Nat. Prod. 1994, 57, 68.

12. Vega, M. R. G.; Souza-Esteves, A.; Vieira, I. J. C.; Mathias, L.; BrazFilho, R.; Echevarria, A.; J. Braz. Chem Soc. 2007, 18, 1554.

13. Moreira, I. C.; Lago, J. H. G.; Young, M. C. M.; Roque, N. F.; J. Braz. Chem. Soc. 2003, 14, 828.

14. Moreira, I. C.; Lago, J. H. G.; Roque, N. F.; Biochem. System. Ecol. 2006, 34, 883.

15. Takahashi, J. A.; Boaventura, M. A. D.; Bayma, J. C.; Oliveira, A. B.; Phytochemistry 1995, 40, 607.

16. Martins, D.; Osshiro, E.; Roque, N. F.; Marks, V.; Gottlieb, H. E.; Phytochemistry 1998, 48, 677.
17. Takahashi, J. A.; Vieira, H. S.; Hanson, J. R.; Hitchcock, P. B.; Oliveira, A. B.; Boaventura, M. A. D.; Quim. Nova 2001, 24, 616; Pontes, W. J. T.; Oliveira, J. C. S.; Câmara, C. A. G.; Gondim-Júnior, M. G. C.; Oliveira, J. V.; Schwartz, M. O. E.; Quim. Nova 2007, 30, 838.

18. Lago, J. H. G.; Chaves, M. H.; Ayres, M. C. C.; Agripino, D. G.; Young, M. C. M.; Planta Med. 2007, 73, 292.

19. Correa, M. P.; Dicionário das Plantas úteis do Brasil, Ministério da Agricultura, Instituto Brasileiro de Desenvolvimento Florestal, 1984, p. 315 .

20. Tavares, J. F.; Queiroga, K. F.; Silva, M. V. B.; Diniz, M. F. F. M.; Barbosa-Filho, J. M.; Da-Cunha, E. V. L.; Simone, C. A.; Araújo-Junior, J. X.; Melo, P. S.; Haun, M.; Silva, M. S.; J. Nat. Prod. 2006, 69, 960.

21. Tavares, J. F.; Silva, M. V. B.; Queiroga, K. F.; Diniz, M. F. F. M.; Barbosa-Filho, J. M.; Haun, M.; Melo, P. S.; Silva, M. S.; Z. Naturforsch. 2007, 62b, 742 .

22. Tavares, J. F.; Silva, M. V. B.; Queiroga, K. F.; Martins, R. M.; Silva, T. M. S.; Camara, C. A.; Agra, M. F.; Barbosa-Filho, J. M.; Da-Silva, M. S.; J. Essent. Oil Res. 2007, 19, 282.

23. Oliveira, A. P.; Furtado, F. F.; Silva, M. S.; Tavares, J. F.; Mafra, R. A.; Araújo, D. A. M.; Cruz, J. F.; Medeiros, I. A.; Vasc. Pharmacol. 2006, $44,338$.

24. Ribeiro, L. A. A.; Tavares, J. F.; Andrade, N. C.; Silva, M. S.; Silva, B. A.; Rev. Bras. Farmacogn. 2007, 17, 197.

25. Leboueuf, M.; Legueut, C.; Cavé, A.; Desconclois, J. F.; Forga, C. S.; Planta Med. 1980, 39, 204; Comins, D. L.; Thakerp, M.; Baevsky, M. F.; Badawi, M. M.; Tetrahedron 1997, 53, 16327.

26. Tourwe, D.; Bisnt, V. G.; Kametani, T.; Organic Magnetic Resonance 1977, 9, 341

27. Jossang, A.; Leboeuf, M.; Cavé, A.; Pusset, J.; J. Nat. Prod. 1991, 54 , 466.

28. Vazquez, M. M.; Lozano, D. G. C.; Estrada-Reyes, R.; Gonzalez-Lugo, N. M.; Apan, T. R.; Heinza, G.; Fitoterapia 2005, 76, 733.

29. Sette, I. M. F.; Da-Cunha, E. V. L.; Barbosa-Filho, J. M.; Silva, M. S.; Pharm. Biol. 2000, 38, 318; Hussain, R. A.; Kim, J.; Beecher, C. W. W.; Kinghorn, A. D.; Heterocycles 1989, 20, 2257.

30. Guinaudeau, H.; J. Nat. Prod. 1983, 46, 763.

31. Leboeuf, M.; Cavé, A.; J. Nat. Prod. 1988, 51, 389.

32. Silva, D. B.; Matos, M. F. C.; Nakashita, S. T.; Misu, C. K.; Yoshida, N. C.; Carollo, C. A.; Fabri, J. R.; Miglio, H. S., Siqueira, J. M.; Quim. Nova 2007, 30, 1809.

33. Pinar, M.; Rico, M.; Rodriguez, B.; Fernandez, B.; Phytochemistry 1984, 23, 195.

34. Schhmitz, F. J.; Vanderah, D. J.; Hollenbeak, K. H.; Enwall, C. E. L.; Gopichand, Y.; Sengupta, P. K.; Hossain, M. B.; Helm, D.; J. Org. Chem. 1983, 48, 3941

35. Moraes, M. P. L.; Roque, N. F.; Phytochemistry 1988, 27, 3205.

36. Silva, D. A.; Silva, T. M. S.; Lins, A. C. S.; Costa, D. A.; Cavalcante, J. M. S.; Matias, W. N.; Souza, M. F. V.; Braz-Filho, R.; Quim. Nova 2006, 29, 1250.

37. Jin, P. F.; Deng, Z. W.; Pei, Y. H.; Lin, W. H.; Chin. Chem. Lett. 2005, 16, 209.

38. Beltrame, F. L.; Sartoretto, J. L.; Bazote, R. B.; Cuman, R. N.; Cortez, D. A. G.; Fernandes, L. C.; Tchaikovski, O.; Quim. Nova 2001, 24, 783.

39. Malterud, K. E.; Farbrot, T. L.; Huse, A. E.; Sund, R. B.; Pharmacology 1993, 47, 77 . 